\title{
The diagnostic value of SPECT/CT in predicting the occurrence of osteonecrosis following femoral neck fracture: a prospective cohort study
}

\author{
Jae Youn Yoon ${ }^{1}$, Soong Joon Lee ${ }^{2}$, Kang Sup Yoon ${ }^{2}$ and Pil Whan Yoon ${ }^{3^{*}}$
}

\begin{abstract}
Backgrounds: One of the most significant complications after a femoral neck fracture is osteonecrosis of the femoral head (ONFH). The concomitant use of single-photon emission computed tomography (SPECT) with computed tomography (CT) increases the sensitivity for detecting the anatomic location and severity of ONFH. In this study, we evaluated the diagnostic value of SPECT/CT for the occurrence of ONFH by quantifying the perfusion status of the femoral head.

Methods: A total of 30 patients who had multiple pinnings for femur neck fractures were included in this study. We classified the perfusion status into three groups: normal perfusion, decreased perfusion, and avascular groups, and compared the occurrence of femoral head necrosis between them. For quantitative analysis, we evaluated the uptake ratio of both femur heads (head-to-head uptake ratio). If the patient's contralateral hip was incomparable, we measured the uptake ratio from the superior dome of the ipsilateral acetabulum (head-to-acetabulum uptake ratio).

Results: Twenty-four patients out of 30 achieved bone union, whereas the others developed ONFH. When the population was divided into intact and defective perfusion groups on scintigraphy, the sensitivity, specificity, and accuracy of the test were $83.3,75.0$, and $76.7 \%$, respectively. The mean head-to-head uptake ratio value with a 95\% confidence interval (Cl) was 1.10 (95\% Cl: 0.85-1.36). In the osteonecrosis group, the mean value of the head-to-head uptake ratio was 0.33 (95\% Cl: 0.28-0.38). In contrast, the ratio was 1.30 (95\% Cl: 1.03-1.57) in the non-osteonecrosis group, demonstrating a significant difference in the uptake ratio $(P<0.001)$. When the cutoff value of the uptake ratio was set to 0.5 , both the sensitivity and specificity were $100 \%$. There was also a significant difference in the head-toacetabulum uptake ratio between the two groups $(P<0.001)$.

Conclusions: SPECT/CT was useful in evaluating the perfusion status of the femoral head, showing high accuracy in predicting the occurrence of avascular necrosis. To demonstrate the reliability and validity of SPECT/CT, further prospective studies on a larger scale are warranted.
\end{abstract}

Keywords: Osteonecrosis, Arthroplasty, replacement, hip, Femur neck fracture, Tomography, emission-computed, single-photon, Tc-99 m-HDP

\footnotetext{
*Correspondence: orthoyoon@amc.seoul.kr

${ }^{3}$ Department of Orthopedic Surgery, University of Ulsan College of Medicine,

Asan Medical Center, 88 Olympic-ro 43-gil, Songpa-gu, Seoul 05505, Republic of Korea

Full list of author information is available at the end of the article
}

C C The Author(s). 2020 Open Access This article is licensed under a Creative Commons Attribution 4.0 International License, which permits use, sharing, adaptation, distribution and reproduction in any medium or format, as long as you give appropriate credit to the original author(s) and the source, provide a link to the Creative Commons licence, and indicate if changes were made. The images or other third party material in this article are included in the article's Creative Commons licence, unless indicated otherwise in a credit line to the material. If material is not included in the article's Creative Commons licence and your intended use is not permitted by statutory regulation or exceeds the permitted use, you will need to obtain permission directly from the copyright holder. To view a copy of this licence, visit http://creativecommons.org/licenses/by/4.0/. The Creative Commons Public Domain Dedication waiver (http://creativecommons.org/publicdomain/zero/1.0/) applies to the data made available in this article, unless otherwise stated in a credit line to the data. 


\section{Background}

The incidence of femoral neck fractures (FNFs) is increasing as the number of elderly people increases. Despite developments in treatment options, FNF still has a high complication rate, which leads to an economic burden to society and necessitates guidelines for successful outcomes [1-3]. The surgical treatment of FNF includes osteosynthesis or arthroplasty, which can be selected based on the displacement of fracture or the age of the patient [4-9]. Several studies reported the incidence of osteonecrosis of the femur head (ONFH) after the osteosynthesis of FNF to be 12.0-17.3\% [7, 10]. Other studies also reported that ONFH occurs in about $9.9-33 \%$ of the displaced fractures and $6.4-10.8 \%$ of the nondisplaced fractures [11-14].

Perfusion failure is considered the primary mechanism responsible for ONFH. Therefore, the exact evaluation of the perfusion status of the femoral head after FNF is critical in predicting the risk of ONFH so that it could guide a surgeon to select the optimal treatment modalities to lower the complication rate and achieve a favorable outcome. To date, various diagnostic methods, including laser Doppler flowmetry, ultrasonography, arterial angiography, intra-osseous venography, intraosseous oxygen pressure measurement, and scintigraphy, have been introduced to assess perfusion status. Among them, scintigraphy is generally considered the modality of choice [15-18]. Scintigraphy has an $87-95 \%$ accuracy in predicting the occurrence of $\mathrm{ONFH}$ or nonunion. However, most of the study results are based on qualitative measurements. Conventional bone scans provide poor image descriptions and have limitations in precisely locating the lesion, which lowers the specificity of the test [16]. Furthermore, the two-dimensionally reconstructed three-dimensional anatomical structure is likely to produce false-negative results due to overlying structures.

Single-photon emission computed tomography (SPEC $\mathrm{T})$ with computed tomography (CT) using a hybrid camera system enables the clinicians to find the exact location of the lesion and assess the severity as well. Several studies also examined the diagnostic accuracy of SPEC T/CT $[17,18]$. Despite the virtues, SPECT/CT still depends on the subjective judgment of the specialists and non-quantitative methods for assessing the image, which diminishes the objectivity and accuracy of the test [19].

To our knowledge, only one study has reported a quantitative analysis of SPECT/CT to predict ONFH in patients with FNF treated with internal fixation [20]. However, their method is only available to patients with FNF with a healthy contralateral hip because they used the photon number ratio in the affected side against the unaffected side. Therefore, we evaluated the diagnostic value of SPECT/CT in assessing the risk of ONFH in patients with FNF. We used three different methods to determine femoral head vascularity: 1) qualitative analysis by specialists board-certified in nuclear medicine, 2) quantitative analysis using the photon uptake ratio in the affected femoral head against the unaffected femoral head, and 3) quantitative analysis using the photon uptake ratio in the affected femoral head against the superior acetabular dome of the affected hip.

\section{Methods \\ Subjects}

The study was a prospective cohort study performed at a single center with 145 patients with FNFs from January 2010 to March 2012 under the approval of the Institutional Review Board. All patients were fully informed of the concept of the study, generally accepted treatment methods and the prognosis of both multiple pinning and arthroplasty before participation in the study. Among the initially enrolled 145 patients, we excluded 90 patients from the study who chose to undergo total hip arthroplasty (THA) or bipolar hemiarthroplasty (BPHA) instead. Of the 52 patients, we also excluded 22 patients due to a lack of preoperative SPECT/CT evaluations and a short follow-up length of less than two years. Finally, 30 patients were enrolled in this study, including 11 men and 19 women with a mean age of 64.3 years (range, 13 to 90 years). The average duration of follow-up was 4.0 years (range, 2.0 to 8.9 years). We used Garden's classification to classify the type of FNFs. There were 17 (56.7\%) Garden I patients; four (13.3\%) Garden II patients; six (20\%) Garden III patients; and three (10\%) Garden IV patients [21].

\section{Surgical technique and postoperative management}

We performed all operations within $48 \mathrm{~h}$ following the initial trauma and surgically treated the patients with closed-reduction and internal fixation using three cannulated screws on a fracture table. The patients maintained partial weight-bearing using crutches or walker assistance for six weeks postoperatively. Full weightbearing was allowed gradually after six weeks. All patients were followed-up in the clinic with serial radiographs at regular intervals (postoperative six weeks; three, six, twelve months, and annually thereafter). Since the screws used to treat fractures are made of stainless steel, we had a limitation in the detection of ONFH using magnetic resonance imaging (MRI). We, therefore, traced the occurrence of ONFH using simple radiographs (total hip anteroposterior \& translateral view) of the patients. All radiographs were analyzed by a boardcertified radiology specialist who was blinded to the SPECT/CT results to detect the occurrence of ONFH. The radiographical diagnostic criteria for ONFH are 1) patch sclerosis and/or subchondral cyst formation, 2) 
crescent sign and/or irregular bone density at femoral head, 3) collapse or flattening of femoral head. We also confirmed the diagnosis of ONFH histopathologically by undergoing bone biopsy of the surgical specimens of patients who underwent total hip arthroplasty (THA) due to ONFH complications.

\section{SPECT/CT evaluation and analysis}

Preoperative SPECT/CTs were obtained within $48 \mathrm{~h}$ of admission to the hospital using a dual-headed gamma camera (Infina, Hawkeye 4, GE Healthcare, Milwaukee, WI, USA) equipped with low-energy high-resolution (LEHR) collimators. The SPECT data were acquired with 60 stops over 180 at $12 \mathrm{~s}$ per stop using a $128 \times 128$ matrix within three to five hours post-injection of 1100 MBq Tc-99 $\mathrm{m}$ HDP. The CT acquisition was performed at $140 \mathrm{kvp}$ and $2.5 \mathrm{~mA}$ using a $512 \times 512$ matrix and a 5 $\mathrm{mm}$ slice thickness (pitch 10, interval $2.95 \mathrm{~mm}$ ). The SPECT images were reconstructed using an iterative algorithm provided by the manufacturer to reduce starring artifacts by bladder activity. And attenuation correction was done with CT-derived attenuation maps.

\section{Qualitative analysis}

For the qualitative analysis, two experienced boardcertified nuclear medicine specialists, who were blinded from the patients' clinical information, classified the perfusion status of the femoral head on the SPECT/CT scans into two groups: the normal perfusion group and the avascular group. Inter-observer discrepancies were resolved through discussion (Fig. 1).

\section{Quantitative analysis: head-to-head uptake ratio}

We used the workstation for quantitative analysis. Scintigraphy images are subject to the amount of injected radionuclide and the variability in its distribution depending on the time interval between the injection and the time point of the test and renal and hepatic function, and differences can be seen even between individuals with normal perfusion [22-24]. Therefore, we tried to compensate for the variability of the measured value by choosing a relative method for the quantitative analysis. We first defined a 3-cm-sized three-dimensional (3-D) circle as the region of interest (ROI), of which the center was aimed at the center of the femoral head, containing maximum cancellous bone without including the cortical bone. Next, we determined the mean photon uptake of the affected femoral head by averaging the photon uptake numbers in the ROI on the coronal, axial, and sagittal planes. The mean photon uptake of the unaffected femoral head was also measured by the same method. Then, the head-to-head uptake ratio was calculated by dividing the value of the affected hip by that of the unaffected hip (Fig. 2).

\section{Quantitative analysis: head-to-acetabulum uptake ratio}

When the presence of metal implants or ONFH at a contralateral hip was confirmed, we substituted the analysis from the head-to-head ratio to the head-toacetabulum ratio by evaluating the photon uptake numbers from the contralateral hip to the ipsilateral acetabular dome. Unlike the femoral head, the acetabular dome is aspherical and surrounded by thicker pelvic cortices, which allows relatively smaller space for applying the ROI. The pre-measured maximum ROI diameter from computed tomography of each patient varied widely from 1.0 to $2.4 \mathrm{~cm}$, depending on their pelvic size and morphology. Therefore, to set the applicable value for all enrolled patients, the ROI at the acetabular dome was defined as a 1-cm-sized 3-D circle containing cancellous bone as much as possible without involving the cortex. The mean photon uptake of the acetabular dome was determined by averaging the photon uptake numbers on the coronal, axial, and sagittal planes (Fig. 3).

\section{Statistical analysis}

Based on the final follow-up, we divided the patients into $\mathrm{ONFH}$ and non-ONFH groups. The sensitivity

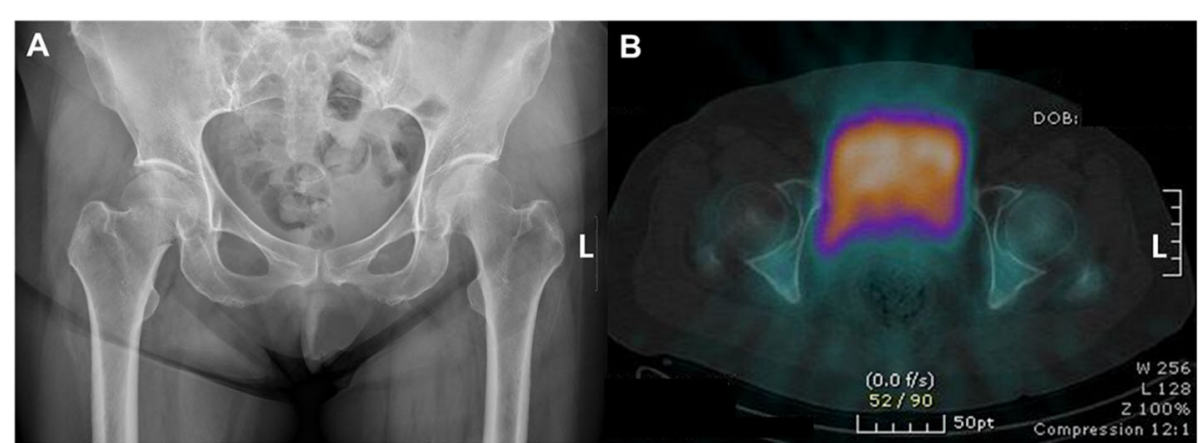

Fig. 1 Qualitative analysis of SPECT/CT. a Right femur neck fracture is diagnosed by simple radiograph. b Photon uptake of the right femur head is decreased compared to the left femur head 


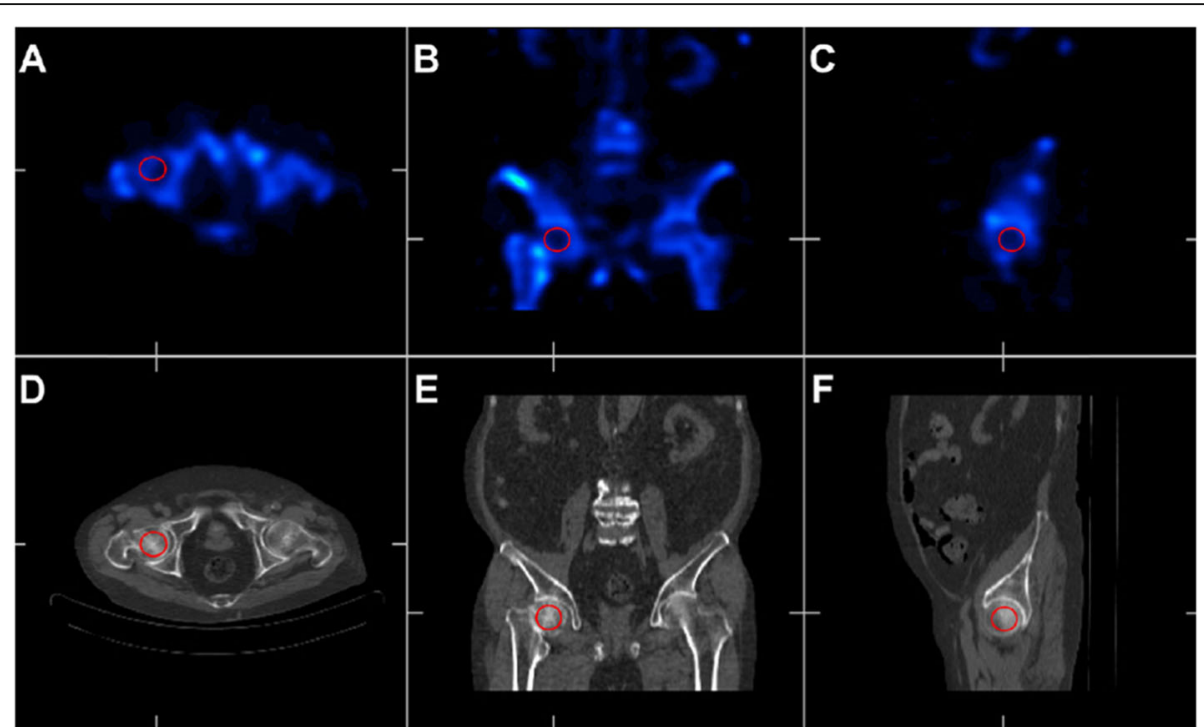

Fig. 2 Quantitative analysis of the head-to-head uptake ratio with ROI in a 3-cm circle, excluding the cortical bone of femur head. a,d ROI measured in axial view of SPECT/CT. b,e ROI in coronal view. c,f ROI in sagittal view. Three independently measured values of pixels are summated to an average value of $\mathrm{ROI}$

and specificity of the qualitative evaluation of the occurrence of osteonecrosis were assessed by crossover analysis. The Mann-Whitney test was used for analyzing numerical data and Fisher's exact test was used for categorical data. A receiver operating characteristics (ROC) curve was plotted from the quantitative analysis data of two groups in order to derive a cut off value for predicting ONFH. Area under curve (AUC) of Two ROC curves were compared using Delong's test for statistical significant change. Statistical analyses were proceeded using SPSS statistical software version 21 (IBM Co., Armonk, NY, USA), and a $P$-value of $<0.05$ was considered statistically significant.

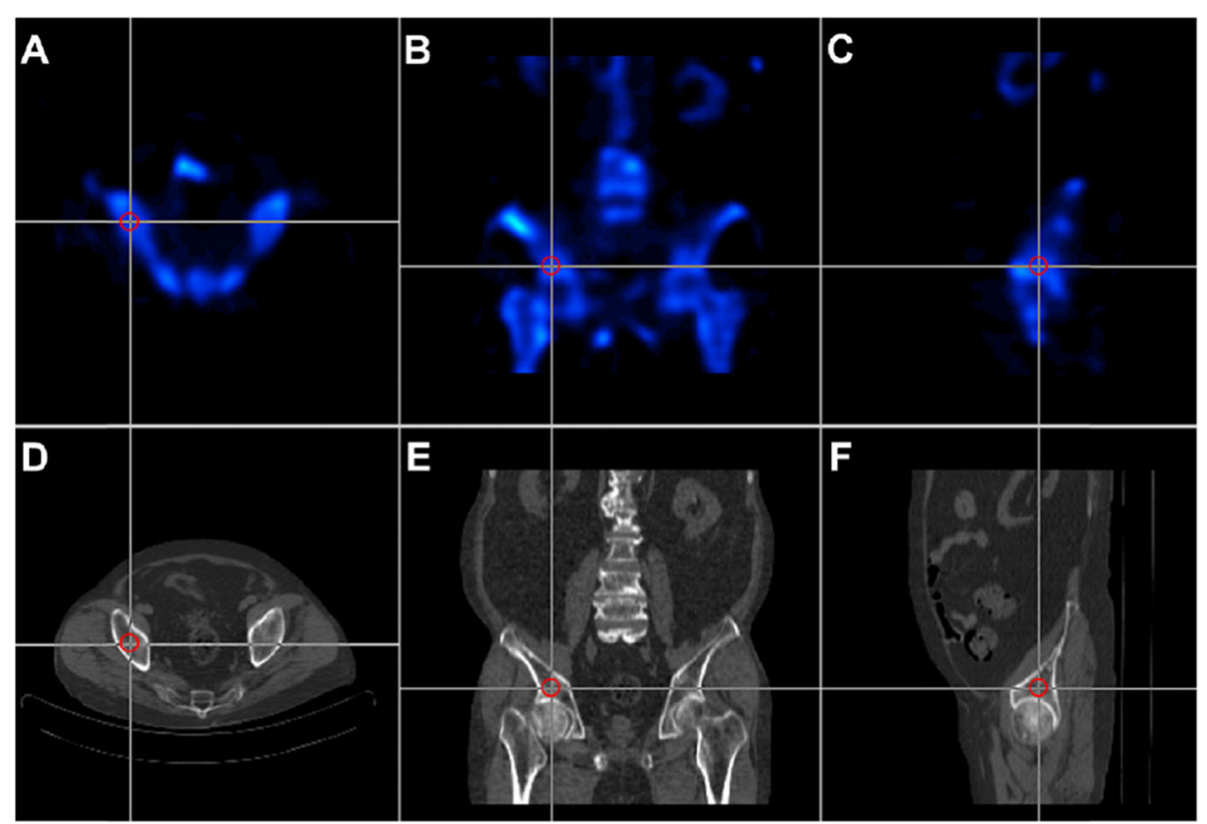

Fig. 3 Quantitative analysis of the head-to-acetabulum uptake ratio with ROI in a 1-cm circle at the dome of the acetabulum in the affected hip. a,d ROI measured in axial view of SPECT/CT. $\mathbf{b}, \mathbf{e}$ ROI in coronal view. c,f ROI in sagittal view. Three independently measured values of pixels are summated to an average value of $\mathrm{ROI}$ 


\section{Results}

At the final follow-up, osteonecrosis developed in six patients out of $30(16.7 \%)$, whereas bone union was confirmed in the other 24 patients without complications. The mean interval of time from surgery to the diagnosis of ONFH was 8.1 months (range, 4.6 to 17 months). The mean age of the ONFH group was significantly younger than that of the non-ONFH group, and the ONFH group also had a higher rate of fracture displacement (Table 1). Other demographic factors showed no significant differences between the groups.

\section{Qualitative evaluation of SPECT/CT}

Eleven patients showed a loss of perfusion, whereas the remaining 19 patients showed normal perfusion. Six out of the 11 patients who showed a loss of perfusion, achieved bone union, whereas the other five patients were diagnosed with ONFH at the final follow-up. Eighteen out of the 19 patients classified into the normal perfusion group achieved bone union, and only one patient was diagnosed with ONFH. The sensitivity, specificity, and diagnostic accuracy of the prediction for the occurrence of osteonecrosis were $83.3,75.0$, and $76.7 \%$, respectively. The positive likelihood ratio and negative likelihood ratio were 3.33 (95\% confidence interval; CI: 1.52-7.27) and 0.22 (95\% CI: 0.04-1.35) (Table 2).

\section{Quantitative evaluation of SPECT/CT}

\section{Evaluation of head-to-head uptake ratio}

At final follow-up, the mean head-to-head uptake ratio of the preoperative SPECT/CT scans was 0.33 (95\% CI, $0.28-0.38$ ) in the ONFH group, whereas the mean uptake ratio was $1.30(95 \% \mathrm{CI}, 1.03-1.57)$ in the nonONFH group, showing a statistically significant difference between the two groups $(p<0.001)$. When ROC curve was plotted to define a best cut off value for predicting the ONFH (Fig. 4), a cutoff value of 0.5 was found to have $100 \%$ sensitivity and specificity for predicting the ONFH (Table 3).

\section{Evaluation of head-to-acetabulum uptake ratio}

Six out of 30 patients had limitations in measuring the existing head-to-head ratio. (status post arthroplasty of the contralateral hip; 4 patients, ONFH of the contralateral hip; 1patient, osteoarthritis of the contralateral hip; 1 patient). The mean uptake ratio of six avascular femoral heads to the ipsilateral acetabular dome was 0.22 (95\% CI: 0.18-0.26), and the mean uptake ratio of 24 intact femoral heads was 0.93 (95\% CI: 0.72-1.14), showing a statistically significant difference $(p<0.001)$. ROC curve was also plotted to define the best cut off value of head-to-acetabulum uptake ratio for predicting the ONFH (Fig. 4). At cut off value of 0.3, the sensitivity and specificity were 85.7 and $100 \%$, respectively (Table 3). When AUC of two ROC curves was compared using DeLong's algorithm, both predicting models showed no statistical difference $(p=0.420)$ [25].

\section{Discussion}

Scintigraphy has its advantage in the early detection of perfusion or metabolism defects before radiologic abnormalities would appear. Scintigraphy can reflect perfusion defects within $24 \mathrm{~h}$ if the test is available and also diagnose and predict $\mathrm{ONFH}$ about 14 months earlier than simple radiography $[19,26]$. Meyers et al. reported a 95\% diagnostic accuracy of scintigraphy in predicting avascular necrosis in his prospective study on FNF patients [27]. Turner et al. also conducted a study with 30 FNF cases using Technetium-99 m scintigraphy and approximated the accuracy of the test at 93\% [28].

The diagnostic accuracy for the qualitative evaluation of ONFH using SPECT in our study was $83.3 \%$ and was suboptimal compared to other studies [27, 28]. Meyer et al., however, included variable indications besides FNF, such as traumatic hip dislocation or idiopathic ischemic necrosis of the femoral head, in assessing diagnostic accuracy [27]. In the results of Turner et al., there were two patients with absent vascular activity in the bilateral femoral head [28]. Depending on the interpretation of these two patients, the diagnostic accuracy may vary from 93 to $86 \%$. In our study, there was one female patient (13-years-old at diagnosis) whose preoperative perfusion status was viable despite the displacement (Garden type 3 at initial diagnosis). Serial clinical and radiological follow-up results showed favorable outcomes but the patient was later diagnosed with ONFH

Table 1 Patient demographics between the two groups

\begin{tabular}{lllll}
\hline Variables (mean, 95\% Cl) & Total $(\mathrm{N}=30)$ & ONFH $(\mathrm{N}=6)$ & Non-ONFH $(\mathrm{N}=24)$ & $p$-value \\
\hline Age (years) & $64.3(57.3-71.3)$ & $44.2(28.3-60.1)$ & $69.3(62.7-75.9)$ & 6.009 \\
Female (\%) & $63.3 \%(n=19)$ & $66.7 \%(n=4)$ & $21.5(20.1-23.0)$ & 0.900 \\
BMI (kg/m2) & $21.8(20.6-23.0)$ & $22.8(20.4-25.2)$ & $0.7(0.6-0.8)$ & 0.273 \\
BMD (g/cm2) & $0.7(0.7-0.8)$ & $0.9(0.7-1.0)$ & $12 \%(n=3)$ & 0.093 \\
Displaced fracture (\%) & $30 \%(n=9)$ & $100 \%(n=6)$ & $2.4(2.3-2.6)$ & 0.001 \\
Duration of follow-up (years) & $2.4(2.2-2.5)$ & $2.1(2.0-2.2)$ & 0.082 \\
\hline
\end{tabular}


Table 2 Sensitivity and specificity for the prediction of the occurrence of ONFH

\begin{tabular}{llll}
\hline Qualitative evaluation & $\begin{array}{l}\text { ONFH } \\
(\boldsymbol{N}=6)\end{array}$ & $\begin{array}{l}\text { Non-ONFH } \\
(\boldsymbol{N}=24)\end{array}$ & Subtotal \\
\hline Decreased or no photon uptake & 5 & 6 & 11 \\
Normal photon uptake & 1 & 18 & 19 \\
Subtotal & 6 & 24 & 30 \\
\hline
\end{tabular}

ONFH osteonecrosis of the femur head

at a postoperative eight-month follow-up. Considering variable clinical factors related to osteonecrosis, such as the patient's age, the degree of displacement, the effect of surgical reduction, and the revascularization potential, the preoperative evaluation of perfusion status should be more complicated. Furthermore, there is still no clear guidelines available for displaced FNF patients, and subjective decisions or the surgeon's preference are used instead. Therefore, a rationale to increase the diagnostic accuracy and positive predictive value of preoperative SPECT/CT in assessing femoral head perfusion status is needed.

Various attempts have been made to predict ONFH by relative quantification. Stromqvist et al. reported that an uptake ratio of the lesion to the unaffected side below 0.9 preoperatively, or 1.0 postoperatively, indicated a high risk of ONFH in displaced FNF patients [29]. Holmberg and Thorngren reported that bone union was expected when the uptake ratio to the unaffected side was over 0.90, whereas complicated outcomes were found under 0.90 [30]. Despite the virtues, conventional planar bone scintigraphy produces poor quality images that cannot provide precise anatomical information, resulting in low specificity. Planar bone scintigraphy also produces a summated 2-dimensional image that can lead to false-negative results by overlying and adjacent bony structures. In this study, therefore, we tried to reveal the predictive value of SPECT/CT based on quantitative analysis of the perfusion status of the femoral head using an ROI.

Quantitative analysis using the head-to-head ratio (with a cutoff value of 0.5 ) in SPECT/CT showed a high diagnostic accuracy of $100 \%$ in predicting osteonecrosis, and the sensitivity and specificity were both $100 \%$. The results were similar to the head-to-acetabulum ratio (with a cutoff value of 0.3 ), showing a diagnostic accuracy of $96.7 \%$, sensitivity and specificity of 85.7 and $100 \%$, respectively. The concept of ROI in quantitative analysis helps to localize the optimal anatomical area for diagnosis and overcome the distortion of the perfusion status. Using the ipsilateral acetabular dome as a reference point for ROI is useful in certain patients whose perfusion status of the contralateral hip is not comparable due to other medication conditions.

There were several limitations to our study. First, the relatively small number of enrolled patients could diminish the reliability of the clinical application of the results. However, our quantitative evaluation results were satisfactory, showing high diagnostic accuracy, as well as

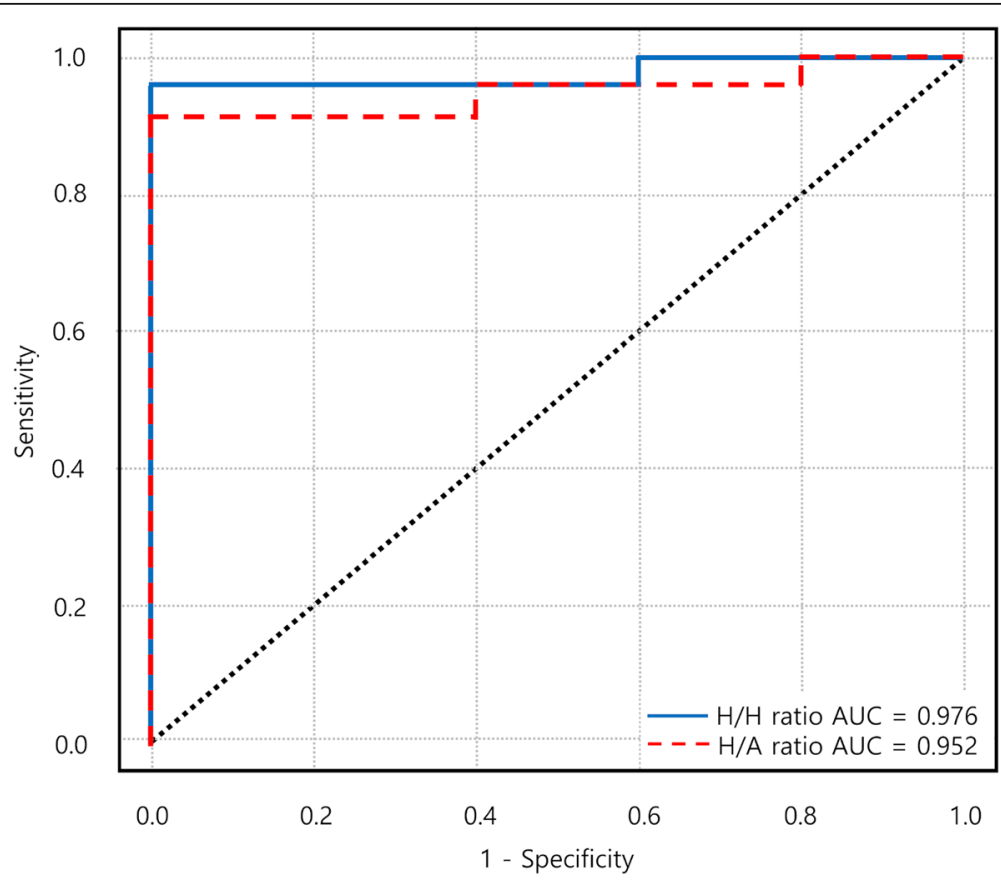

Fig. 4 ROC curve plotted from the data of each group. The head-to-head ratio of 0.5 and the head-to-acetabulum ratio of 0.3 is most sensitive for predicting the occurrence of ONFH 
Table 3 Comparison of the head-to-head ratio and head-to-acetabulum ratio between two groups

\begin{tabular}{llll}
\hline & ONFH $(n=6)$ & Non-ONFH $(n=24)$ & $p$-value \\
\hline Uptake of femoral head & & & $247.6(134.9-360.3)$ \\
$\quad$ Affected hip & $66.3(53.7-79.0)$ & $206.6130 .9-282.3)$ & 0.044 \\
Unaffected hip & $201.3(172.3-230.3)$ & & 0.631 \\
Uptake of acetabulum & & $285.7(182.9-388.7)$ & 0.561 \\
Affected hip & $319.1(219.6-418.6)$ & $290.2(194.1-386.4)$ & 0.527 \\
Unaffected hip & $337.2(217.2-457.2)$ & $1.30(1.03-1.57)$ & $<0.001$ \\
Head-to-head ratio & $0.33(0.28-0.38)$ & $0.93(0.72-1.14)$ & $<0.001$ \\
Head-to-acetabulum ratio & $0.22(0.18-0.26)$ & &
\end{tabular}

ONFH osteonecrosis of the femur head

sensitivity and specificity. The follow-up duration was also sufficient enough to detect most of the complications related to osteonecrosis. Second, postoperative follow-up with SPECT/CT was not conducted. Several studies reported the optimal timing of the test at two weeks after surgery, as revascularization, particularly after fracture reduction, is maximized at that time [31-33]. We tried to take SPEC T/CT scans two weeks and six months postoperatively, but overcorrection related to implants in the attenuation correction process made the analysis impossible. If further studies identify a solution to the problem of attenuation correction, revascularization and changes in perfusion, with or without reduction, as well as their effects on the occurrence of avascular necrosis, could be revealed. Third, the prediction of ONFH was solely based on the preoperative perfusion state. Various factors affecting osteonecrosis, including patient age, the degree of fracture displacement, and the quality of reduction, should be analyzed. Compared to a study by Turner et al., in which half of the patients were Garden type 3 (50\%) fractures, type 1 fractures (56.7\%) outnumbered other types of fractures in our study [28]. Since the displacement is minimal, it is hard to predict the perfusion status both quantitatively and qualitatively. Finally, the diagnosis of postoperative ONFH could be underestimated since we only used a simple radiograph to diagnose the occurrence of postoperative ONFH. To overcome this limitation, however, we have radiologically evaluated the patients for a relatively long time with a mean follow-up of 4 years (range, 2.0 to 8.9 years) and also confirmed the diagnosis by bone biopsy of the retrieved femoral head. Since it is widely accepted that ONFH can occur up to 2-3 years after surgery, our longer follow-up would be sufficient to offset this limitation [34].

\section{Conclusions}

Quantitative evaluation of the perfusion status of the femoral head with SPECT/CT improved the diagnostic accuracy in predicting ONFH and could help determine the optimal surgical method and reduce postoperative complications, including reoperation or conversion to arthroplasty. We suggest a cutoff value of 0.5 in the head-to-head ratio and 0.3 in the head-to-acetabular dome ratio as predictive values of post-traumatic ONFH.

\section{Supplementary information}

Supplementary information accompanies this paper at https://doi.org/10. 1186/s12891-020-03538-1.

\section{Additional file 1.}

\section{Acknowledgements}

Not applicable.

\section{Authors' contributions}

JYY is responsible for writing the whole manuscript, organizing data and analyzing it statistically. SJL and KSY is responsible for collecting patients,organizing the data, and actually conducting the research. PHY is respondible for designing the concept of the study and actually taking responsibility for manuscript correction as well as investigating references, as a corresponding author. All authors read and approved the final manuscript.

\section{Funding}

There is no funding source.

\section{Availability of data and materials}

The datasets used and analyzed during the current study are presented as a supplementary file.

\section{Ethics approval and consent to participate}

This is a prospective cohort study, and was conducted under the approval of Seoul Metropolotan Government-Seoul National University Boramae Medical Center Insituitional review board (SMG-SNU BMC IRB; IRB No. 20100729 / 062010-114/98). Patient's informed consent was signed in a written format.

\section{Consent for publication}

A written format of informed consent, which includes information on the use, disclosure and publication of patient information on the condition of anonymity, was signed and obtained from all individual participants in the study

\section{Competing interests}

The authors declare that they have no competing interests.

\section{Author details}

'Department of Orthopedic Surgery, Dongguk University Ilsan Hospital, Gyeonggi-do, Republic of Korea. ${ }^{2}$ Department of Orthopedic Surgery, 
SMG-SNU Boramae Medical Center, Seoul National University College of Medicine, Seoul, Republic of Korea. ${ }^{3}$ Department of Orthopedic Surgery, University of Ulsan College of Medicine, Asan Medical Center, 88 Olympic-ro 43-gil, Songpa-gu, Seoul 05505, Republic of Korea.

Received: 21 April 2020 Accepted: 23 July 2020

Published online: 03 August 2020

\section{References}

1. Haleem S, Lutchman L, Mayahi R, Grice JE, Parker MJ. Mortality following hip fracture: trends and geographical variations over the last 40 years. Injury. 2008;39:1157-63.

2. Wehren LE, Magaziner J. Hip fracture: risk factors and outcomes. Curr Osteoporos Rep. 2003;1:78-85.

3. Samelson EJ, Zhang YQ, Kiel DP, Hannan MT, Felson DT. Effect of birth cohort on risk of hip fracture: age-specific incidence rates in the Framingham study. Am J Public Health. 2002;92:858-62.

4. Bhandari M, Devereaux PJ, Tornetta P, et al. Operative management of displaced femoral neck fractures in elderly patients - An international survey. J Bone Joint Surg Am. 2005;87a:2122-30.

5. Shah AK, Eissler J, Radomisli T. Algorithms for the treatment of femoral neck fractures. Clin Orthop Relat Res. 2002;399:28-34.

6. Iorio R, Schwartz B, Macaulay W, Teeney SM, Healy WL, York S. Surgica treatment of displaced femoral neck fractures in the elderly - a survey of the American Association of hip and Knee Surgeons. J Arthroplast. 2006;21: 1124-33.

7. Rogmark C, Carlsson A, Johnell O, Sernbo I. A prospective randomised trial of internal fixation versus arthroplasty for displaced fractures of the neck of the femur - Functional outcome for 450 patients at two years. J Bone Joint Surg Br. 2002;84b:183-8.

8. Gjertsen JE, Vinje T, Engesaeter LB, et al. Internal Screw Fixation Compared with Bipolar Hemiarthroplasty for Treatment of Displaced Femoral Neck Fractures in Elderly Patients. J Bone Joint Surg Am. 2010;92a:619-28.

9. Massie WK. Treatment of femoral neck fractures emphasizing long term follow-up observations on aseptic necrosis. Clin Orthop Relat Res. 1973;92: 16-62.

10. Ghayoumi P, Kandemir U, Morshed S. Evidence based update: open versus closed reduction. Injury. 2015;46:467-73.

11. Skinner P, Riley D, Ellery J, Beaumont A, Coumine R, Shafighian B. Displaced subcapital fractures of the femur: a prospective randomized comparison of internal fixation, hemiarthroplasty and total hip replacement. Injury. 1989;20: 291-3.

12. Sikorski JM, Barrington R. Internal fixation versus hemiarthroplasty for the displaced subcapital fracture of the femur. A prospective randomised study. J Bone Joint Surg Br. 1981;63:357-61.

13. Mussbichler $\mathrm{H}$. Arteriographic findings in patients with degenerative osteoarthritis of the hip. Radiology. 1973;107:21-7.

14. Slobogean GP, Sprague SA, Scott T, Bhandari M. Complications following young femoral neck fractures. Injury. 2015;46:484-91.

15. Subramanian G, McAfee JG, Blair RJ, Kallfelz FA, Thomas FD. Technetium99m-methylene diphosphonate-a superior agent for skeletal imaging: comparison with other technetium complexes. J Nucl Med. 1975;16:744-55.

16. Linke R, Kuwert T, Uder M, Forst R, Wuest W. Skeletal SPECT/CT of the peripheral extremities. AJR Am J Roentgenol. 2010;194:W329-35.

17. Römer W, Nömayr A, Uder M, Bautz W, Kuwert T. SPECT-guided CT for evaluating foci of increased bone metabolism classified as indeterminate on SPECT in cancer patients. J Nucl Med. 2006;47:1102-6.

18. Mariani G, Bruselli L, Kuwert T, et al. A review on the clinical uses of SPECT/ CT. Eur J Nucl Med Mol I. 2010:37:1959-85.

19. Ehlinger M, Moser T, Adam P, et al. Early prediction of femoral head avascular necrosis following neck fracture. Orthop Traumatol Surg Res. 2011; 97:79-88.

20. Yuan HF, Shen F, Zhang J, Shi HC, Gu YS, Yan ZQ. Predictive value of single photon emission computerized tomography and computerized tomography in osteonecrosis after femoral neck fracture: a prospective study. Int Orthop. 2015;39:1417-22.

21. Garden RS. Low-angle fixation in fractures of the femoral neck. J Bone Joint Surg Br. 1961;43:647-61.

22. McAfee JG, Singh A, Roskopf M, et al. Experimental drug-induced changes in renal function and biodistribution of 99mTc-MDP. Investig Radiol. 1983; 18:470-8.
23. Fogelman I, Collier BD, Brown ML. Bone scintigraphy: part 3. Bone scanning in metabolic bone disease. J Nucl Med. 1993;34:2247-52

24. Hung JC, Ponto JA, Hammes RJ. Radiopharmaceutical-related pitfalls and artifacts. Semin Nucl Med. 1996:26:208-55.

25. DeLong ER, DeLong DM, Clarke-Pearson DL. Comparing the areas under two or more correlated receiver operating characteristic curves: a nonparametric approach. Biometrics. 1988;44:837-45.

26. Greiff J. Determination of the vitality of the femoral head with $99 \mathrm{mTc}-\mathrm{Sn}$ pyrophosphate scintigraphy. Acta Orthop Scand. 1980;51:109-17.

27. Meyers MH, Telfer N, Moore TM. Determination of the vascularity of the femoral head with technetium 99m-Sulphur-colloid. J Bone Joint Surg Am. 1977;59:658-64.

28. Turner JH. Post-traumatic avascular necrosis of the femoral head predicted by preoperative technetium-99m antimony-colloid scan. An experimental and clinical study. J Bone Joint Surg Am. 1983:65:786-96.

29. Stromqvist B, Hansson LI, Ljung P, Ohlin P, Roos H. Preoperative and postoperative Scintimetry after femoral-neck fracture. J Bone Joint Surg Br. 1984;66:49-54.

30. Holmberg S, Thorngren KG. Preoperative $99 \mathrm{mTc}-\mathrm{MDP}$ scintimetry of femoral neck fractures. Acta Orthop Scand. 1984;55:430-5.

31. Amarasekera HW, Costa ML, Parsons $\mathrm{N}$, et al. SPECT/CT bone imaging after hip resurfacing arthroplasty: is it feasible to use $C T$ attenuation correction in the presence of metal implants? Nucl Med Commun. 2011;32:289-97.

32. Schulz V, Nickel I, Nomayr A, et al. Effect of CT-based attenuation correction on uptake ratios in skeletal SPECT. Nuklearmedizin. 2007;46:36-42.

33. Patton JA, Turkington TG. SPECT/CT physical principles and attenuation correction. J Nucl Med Technol. 2008;36:1-10.

34. Barnes R, Brown JT, Garden RS, Nicoll EA. Subcapital fractures of the femur. A prospective review. J Bone Joint Surg (Br). 1976;58:2-24.

\section{Publisher's Note}

Springer Nature remains neutral with regard to jurisdictional claims in published maps and institutional affiliations.
Ready to submit your research? Choose BMC and benefit from:

- fast, convenient online submission

- thorough peer review by experienced researchers in your field

- rapid publication on acceptance

- support for research data, including large and complex data types

- gold Open Access which fosters wider collaboration and increased citations

- maximum visibility for your research: over $100 \mathrm{M}$ website views per year

At BMC, research is always in progress.

Learn more biomedcentral.com/submissions 